Density Functional Theory Studies on Copper Phenanthroline Complexes. Arturo Robertazzi, ${ }^{a}$ Alessandra Magistrato, ${ }^{* a}$ Paul de Hoog, ${ }^{b}$ Paolo Carloni, ${ }^{a}$ and Jan Reedijk.

${ }^{a}$ International School for Advanced Studies and CNR-INFM-Democritos National Simulation Center, Trieste, Italy

${ }^{\mathrm{b}}$ Leiden Institute of Chemistry, Gorlaeus Laboratories, Leiden University, P.O. Box 9502, 2300 RA Leiden, The Netherlands

Supporting Information Available: Test calculations; Tables 1S and 2S contain comparison between calculated and experimental geometrical parameters of copper complexes; Tables $3 \mathrm{~S}$ and $4 \mathrm{~S}$ report $\mathrm{Cu}-$ hydroxyl complexes bonding parameters; Figures 1S-4S. This material is available free of charge via the Internet at http://pubs.acs.org.

\title{
Test calculations on $\mathrm{Cu}(\text { phen })_{2}{ }^{+}, \mathrm{Cu}(\text { phen })_{2}{ }^{2+}$ and $\mathrm{Cu}(\text { phen })_{2}\left(\mathrm{H}_{2} \mathrm{O}\right)^{2+}$
}

In order to test the accuracy of our calculation set-up based on DFT-BLYP, we have optimised structures of $\mathrm{Cu}(\text { phen })_{2}{ }^{+}, \mathrm{Cu}(\text { phen })_{2}{ }^{2+}$ and $\mathrm{Cu}(\text { phen })_{2}\left(\mathrm{H}_{2} \mathrm{O}\right)^{2+}$, and compared them with the available experimental and theoretical data. ${ }^{1-7}$ In particular, structures of $\mathrm{Cu}(\mathrm{phen})_{2}{ }^{2+}$ optimised with the BLYP functional are in very good agreement with previous theoretical studies. ${ }^{8}$

Moreover, our optimised geometry of $\mathrm{Cu}(\mathrm{phen})_{2}\left(\mathrm{H}_{2} \mathrm{O}\right)^{2+}$ is compared to the experimental one of several crystals with different counter ions, Tab. 1S. ${ }^{9}$ DFT performance is fairly good, although Cu-ligand distances are generally out of the experimental range. This may be due to the strong counter ion dependence showed by experimental values.

The available experimental structural data most similar to $\mathrm{Cu}(\text { phen })_{2}{ }^{+}$are those of $\mathrm{Cu}(\mathrm{I})$-2,9-dimethyl1,10-phenanthroline or $\mathrm{Cu}(\mathrm{dmp})_{2}{ }^{+10}$ Following previous DFT studies ${ }^{2,6}$ we have compared the structural determinants of our $\mathrm{Cu}(\mathrm{phen})_{2}{ }^{+}$with those of $\mathrm{Cu}(\mathrm{dmp})_{2}{ }^{+10},{ }^{10}$ under the reasonable assumption that the effect of methyl groups is negligible (Fig. 1S). An experimental study that explores the effect of counter ions on the structure of reduced $\mathrm{Cu}(\mathrm{phen})_{2}$ is not available, however little effect should be expected because of smaller charge on $\mathrm{Cu}$. Nevertheless, calculated bond lengths and angles differ from experimental ones by less than $0.1 \AA$ and $4^{\circ}$, respectively, with the largest difference being the dihedral angle between aromatic planes $\left(\sim 10^{\circ}\right)$. These small differences may be due to intermolecular forces in the crystal, such as strong $\pi$-stack interactions, that may become less important in solution. 
For instance, NMR studies at room temperature indicate the two phenanthroline ligands to be equivalent, i.e., confirming a higher symmetry in solution than that determined in crystals. ${ }^{11}$

In addition, a few DFT studies were performed on these complexes, leading to a satisfactory agreement between theory and experiment. ${ }^{2,3,5-7,12}$ Therefore, we conclude that the DFT calculations provide a fair description of these complexes.

One of the aims of this study is to characterise structures of $\mathrm{Cu}$ (2-Clip-phen) and $\mathrm{Cu}$ (3-Clip-phen) complexes in both reduced and oxidized states. As shown by Pitié et al., ${ }^{13}$ crystallization of compounds with clipped ligands was rather difficult and the only experimental data available refer to "open" dimers including two metals centres and four phenanthroline rings. Therefore, we have chosen an alternative route, testing structures optimised at DFT-BLYP level with those obtained with the B3LYP functional, employing different basis sets (from 6-31G(d), 6-31+G(d,p) to 6-311++G(d,p)). Tab. 2S shows that geometries of $\mathrm{Cu}(3-\mathrm{Clip}-\text { phen })^{+}$are equivalent and virtually independent from the functional and the basis set. For instance $\mathrm{Cu}-\mathrm{N}$ bonds calculated at BLYP/6-31G(d) differ by $\Delta \mathrm{d} \sim 0.03 \AA$ from B3LYP/6-311++G(d,p). Likewise, angles and dihedral angles between phenanthroline rings differ by less than $1^{\circ}$, on average. Similar results were found for all other clipped complexes (data not shown).
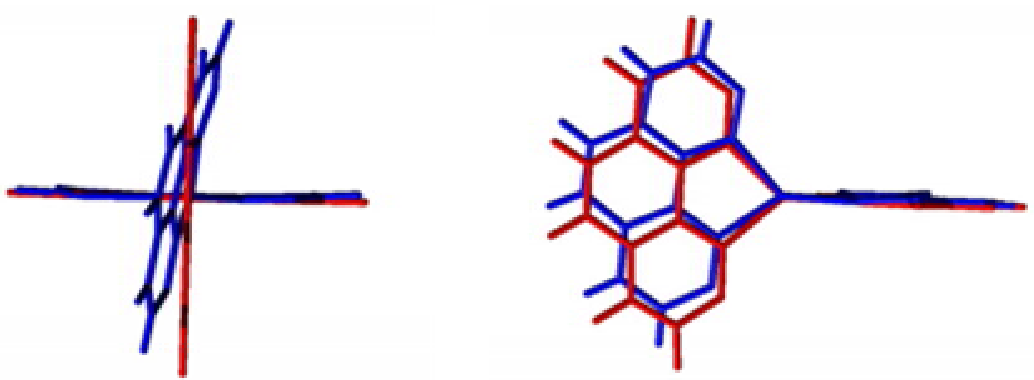

Figure 1S. Comparison between experimental structure (blue $)^{10}$ of $\mathrm{Cu}(\mathrm{dmp})_{2}{ }^{+}$, and optimised structure of $\mathrm{Cu}(\mathrm{phen})_{2}{ }^{+}$(red). For the sake of clarity, methyl groups of $\mathrm{Cu}(\mathrm{dmp})_{2}{ }^{+}$are not shown. 


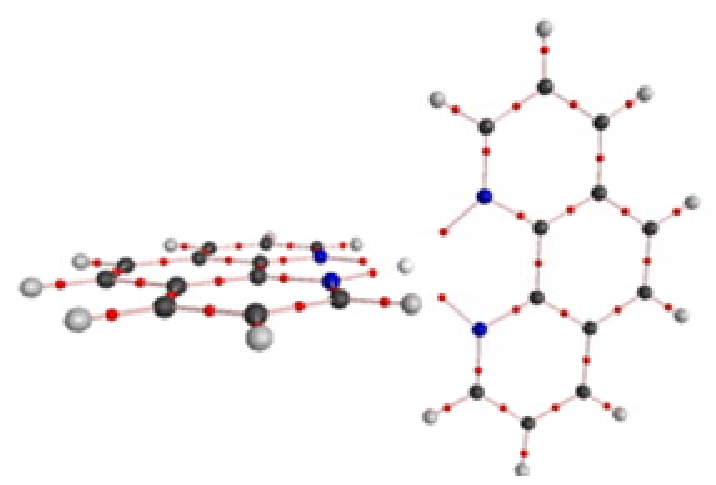

$\mathrm{Cu}(\text { phen })_{2}^{+}$

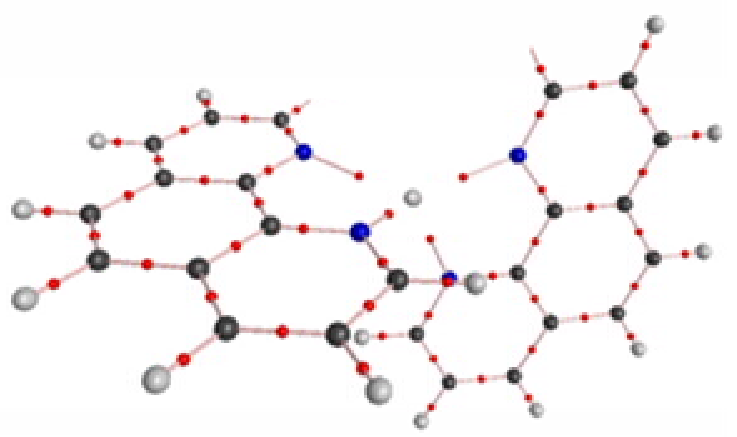

$\mathrm{Cu}$ (2-Clip-phen $)^{+}$

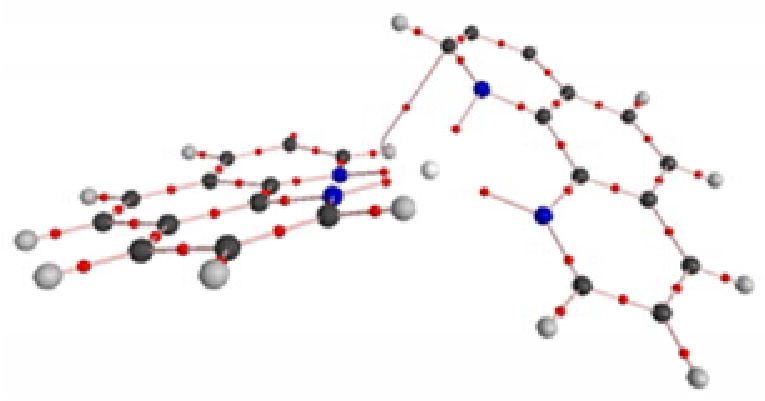

Cu(3-Clip-phen) ${ }^{+}$

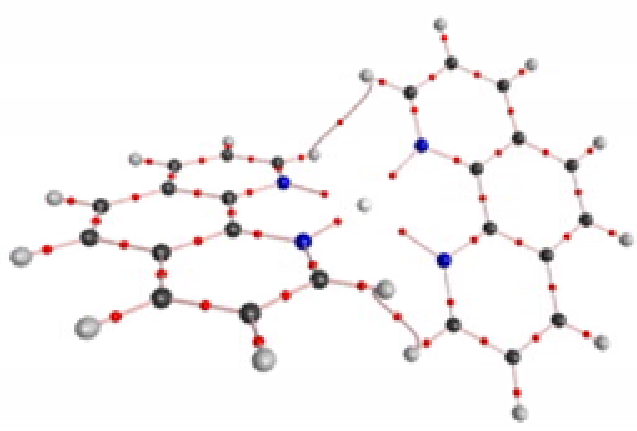

$\mathrm{Cu}(\text { phen })_{2}{ }^{2+}$

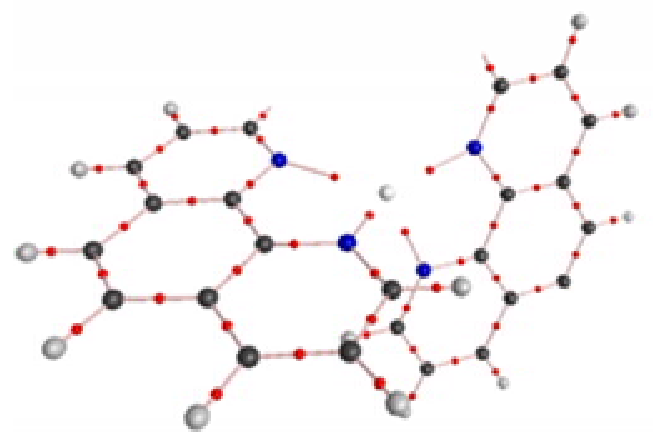

$\mathrm{Cu}$ (2-Clip-phen $)^{2+}$

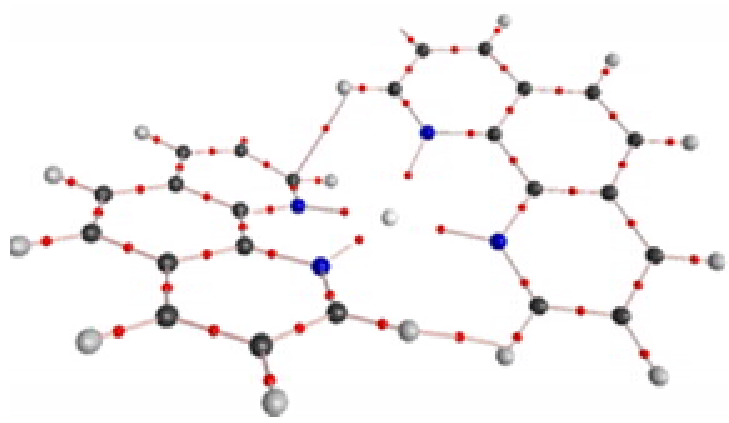

$\mathrm{Cu}$ (3-Clip-phen) ${ }^{2+}$

Figure 2S. AIM topologies of $\mathrm{Cu}(\text { phen })_{2}{ }^{+, 2+}, \mathrm{Cu}\left(2\right.$-Clip-phen) ${ }^{+, 2+}$ and $\mathrm{Cu}\left(3-\mathrm{Clip}-\mathrm{Cu}\right.$-phen) ${ }^{+, 2+}$ complexes (serinol links are not shown for clarity). 

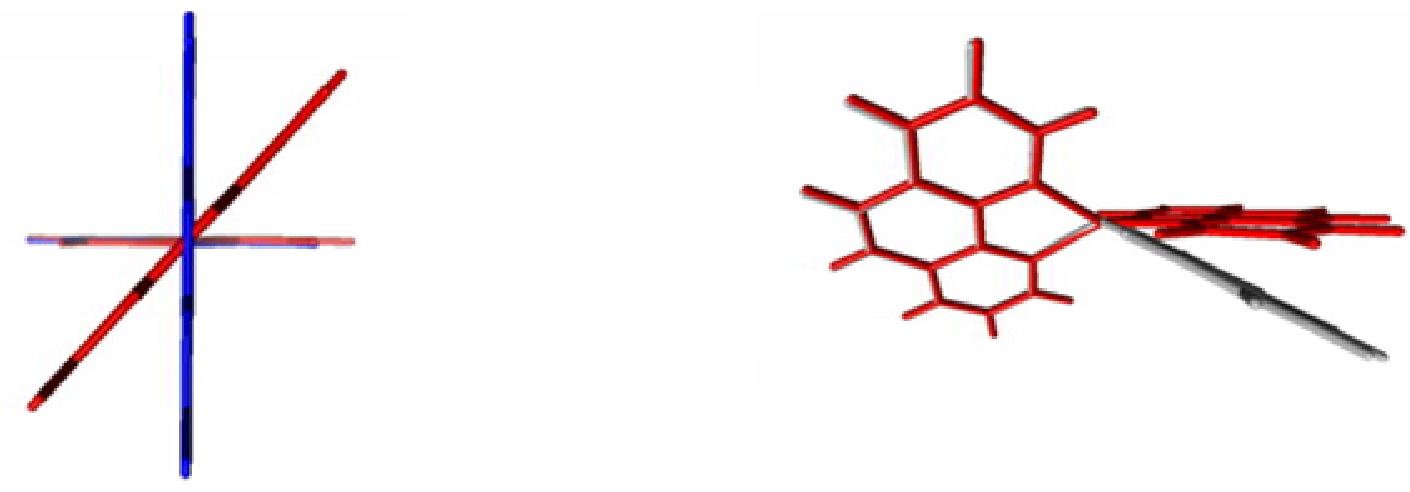

Figure 3S. Superposition of $\mathrm{Cu}(\text { phen })_{2}{ }^{+}$(blue), $\mathrm{Cu}$ (phen $)_{2}{ }^{2+}$ (red) and $\mathrm{Cu}(\text { phen })_{2}\left(\mathrm{H}_{2} \mathrm{O}\right)^{2+}$ (grey) (water molecule is not shown for clarity).
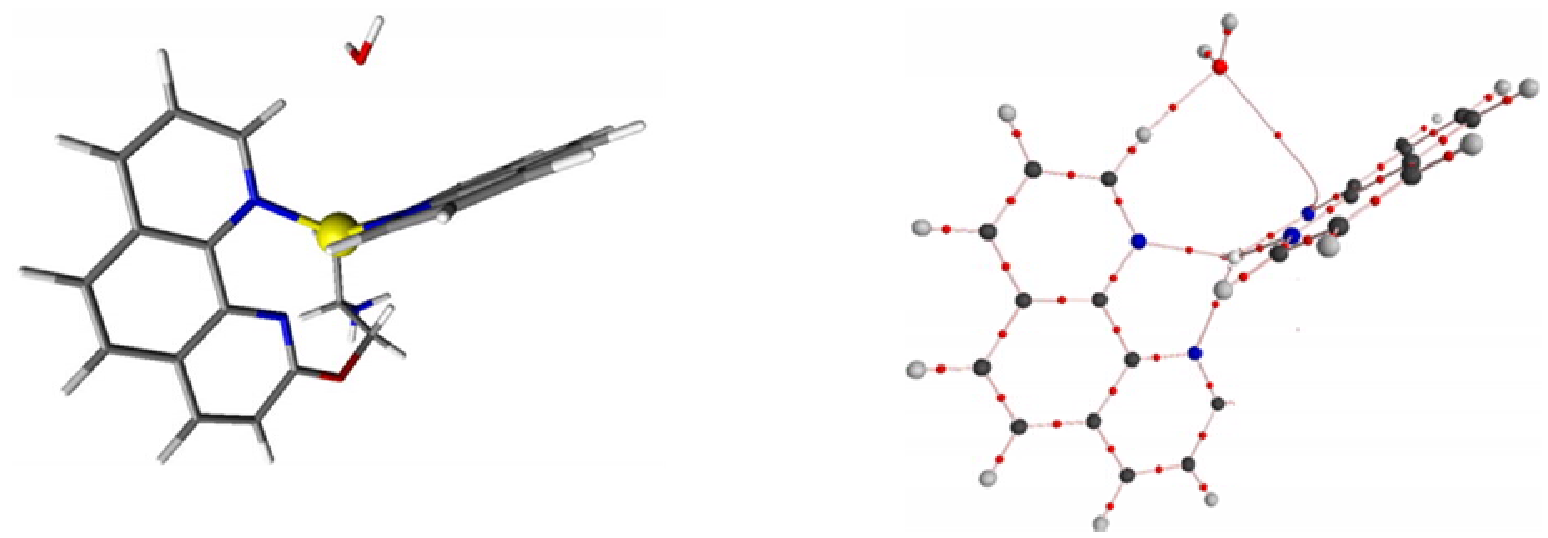

Figure 4S. Geometry and topology (serinol link is not shown for clarity) of the $\mathrm{Cu}\left(2\right.$-Clip-phen) ${ }^{2+} \ldots \mathrm{H}_{2} \mathrm{O}$ adduct (see text for further details). 
Table 1S. Comparison between experimental and calculated parameters of $\mathrm{Cu}\left(\mathrm{H}_{2} \mathrm{O}\right)(\mathrm{phen})_{2}{ }^{2+}$ (see Fig. 1 for atom labelling scheme). Parameters with small dependence on the nature of counterions are shown in bold.

\begin{tabular}{lll}
\hline & Expt. $^{9}$ range & BLYP/6-31G(d) \\
\hline Cu-O & $1.982-2.245$ & 2.31 \\
Cu1-N1 & $\mathbf{1 . 9 7 3 - 2 . 0 0 0}$ & $\mathbf{2 . 0 4}$ \\
Cu1-N2 & $\mathbf{2 . 0 1 3 - 2 . 1 0 0}$ & $\mathbf{2 . 1 1}$ \\
N2-Cu-OH 2 & $110.2-143.6$ & 122.3 \\
N4-Cu-N2 & $99.8-139.6$ & 131.6 \\
N1-Cu-OH & $\mathbf{8 5 . 8 - 9 2 . 3}$ & $\mathbf{8 5 . 3}$ \\
N2-Cu-N1 & $\mathbf{7 9 . 8 - 8 2 . 6}$ & $\mathbf{8 1 . 3}$ \\
N3-Cu-N1 & $\mathbf{1 7 4 . 6 - 1 7 9 . 1}$ & $\mathbf{1 7 5 . 0}$ \\
N3-Cu-N2 & $\mathbf{9 6 . 5 - 1 0 0 . 9}$ & $\mathbf{1 0 0 . 1}$ \\
\hline
\end{tabular}

Table 2S. Comparison between selected bond lengths and angles structures of $\mathrm{Cu}(3-\mathrm{Clip}-\mathrm{phen})^{+}$optimized at the BLYP and B3LYP level (see text for further details).

\begin{tabular}{lc|ccc}
\hline & $\begin{array}{c}\text { BLYP } \\
6-31 G(d)\end{array}$ & $6-31 G(d)$ & $6-31+G(d, p)$ & $6-311++G(d, p)$ \\
\hline Cu-N & 2.14 & 2.19 & 2.18 & 2.18 \\
& 2.14 & 2.19 & 2.18 & 2.18 \\
& 2.06 & 2.06 & 2.05 & 2.05 \\
& 2.07 & 2.06 & 2.06 & 2.06 \\
N-C & 1.47 & 1.46 & 1.46 & 1.46 \\
N1-Cu-N2 & 80.6 & 79.5 & 79.7 & 79.6 \\
& 80.7 & 79.4 & 79.6 & 79.5 \\
N1-N2-N3-N4 & 49.4 & 49.4 & 49.9 & 50.0 \\
\hline
\end{tabular}


Table 3S. Selected bond lengths $(\AA)$, angles and dihedral angles (deg) of $\mathrm{Cu}(\text { phen })_{2}+, 2+, \mathrm{Cu}(2 \text {-Clip-phen })^{+, 2+}$ and $\mathrm{Cu}(3-\mathrm{Clip}-\text { phen })^{+, 2+}$ adducts with water and hydroxyl complexes.

\begin{tabular}{|c|c|c|c|c|c|c|c|c|}
\hline $\begin{array}{l}\text { Oxidation } \\
\text { State }\end{array}$ & Ligand(s) & $\mathrm{Cu}-\mathrm{N} 1$ & $\mathrm{Cu}-\mathrm{N} 2$ & $\mathrm{Cu}-\mathrm{N} 3$ & $\mathrm{Cu}-\mathrm{N} 4$ & $\begin{array}{c}\mathrm{Cu}-\mathrm{X} \\
\left(\mathrm{X}=\mathrm{H}_{2} \mathrm{O}, \mathrm{OH},\right. \\
\left.\mathrm{OH}^{-}\right)\end{array}$ & $\mathrm{N} 1-\mathrm{Cu}-\mathrm{N} 3$ & N1-N2-N3-N4 \\
\hline \multirow[t]{3}{*}{$\mathrm{Cu}(\mathrm{II})$} & $\left.(\text { phen })_{2}\right)\left(\mathrm{H}_{2} \mathrm{O}\right)$ & 2.04 & 2.11 & 2.04 & 2.12 & 2.31 & 175 & 40 \\
\hline & (2-Clip-phen) $\left(\mathrm{H}_{2} \mathrm{O}\right)$ & 2.09 & 2.07 & 2.01 & 2.45 & 2.45 & 161 & 70 \\
\hline & (3-Clip-phen) $\left(\mathrm{H}_{2} \mathrm{O}\right)$ & 2.12 & 2.06 & 2.11 & 2.09 & 2.33 & 168 & 33 \\
\hline \multirow[t]{3}{*}{$\mathrm{Cu}(\mathrm{II})$} & $\left.(\text { phen })_{2}\right)(\mathrm{OH})$ & 2.12 & 2.37 & 2.08 & 2.15 & 1.90 & 167 & 63 \\
\hline & $(2-C l i p-p h e n)(\mathrm{OH})$ & 2.15 & 2.07 & 2.09 & 2.88 & 1.90 & 176 & 74 \\
\hline & (3-Clip-phen) $(\mathrm{OH})$ & 2.18 & 2.30 & 2.09 & 2.18 & 1.88 & 158 & 51 \\
\hline \multirow[t]{3}{*}{$\mathrm{Cu}(\mathrm{II})$} & $\left.(\text { phen })_{2}\right)(\mathrm{OH})^{*}$ & 1.98 & 2.36 & 1.98 & 2.01 & 1.83 & 167 & 62 \\
\hline & $(2$-Clip-phen $)(\mathrm{OH})^{*}$ & 1.93 & 1.98 & 1.91 & 2.67 & 1.83 & 175 & 75 \\
\hline & $(3-C l i p-p h e n)(\mathrm{OH}) *$ & 2.02 & 2.32 & 2.00 & 2.02 & 1.84 & 161 & 53 \\
\hline
\end{tabular}

${ }^{*}$ copper complexes with the radical $\mathrm{OH}^{*}$

Table 4S. Electron density and $\nabla^{2} \rho$ at $\mathrm{Cu}-\mathrm{N}$ and $\mathrm{Cu}-\mathrm{OH}_{2}($ or $\mathrm{Cu}-\mathrm{OH})$ Bond Critical Points of $\mathrm{Cu}(\text { phen })_{2}{ }^{+, 2+}, \mathrm{Cu}(2-\mathrm{Clip}-\mathrm{phen})^{+, 2+}$ and $\mathrm{Cu}(3-\text {-Clip-phen })^{+, 2+}$ adducts with water and hydroxyl complexes.

\begin{tabular}{|c|c|c|c|c|c|c|c|c|c|c|c|}
\hline \multirow[b]{2}{*}{$\begin{array}{c}\text { Oxidation } \\
\text { State }\end{array}$} & \multirow[b]{2}{*}{ Ligand(s) } & \multicolumn{2}{|c|}{$\mathrm{Cu}-\mathrm{N} 1$} & \multicolumn{2}{|c|}{$\mathrm{Cu}-\mathrm{N} 2$} & \multicolumn{2}{|c|}{$\mathrm{Cu}-\mathrm{N} 3$} & \multicolumn{2}{|c|}{$\mathrm{Cu}-\mathrm{N} 4$} & \multicolumn{2}{|c|}{$\mathrm{Cu}-\mathrm{X}\left(\mathrm{X}=\mathrm{H}_{2} \mathrm{O}, \mathrm{OH}, \mathrm{OH}^{-}\right)$} \\
\hline & & $\rho$ & $\nabla^{2} \rho$ & $\rho$ & $\nabla^{2} \rho$ & $\rho$ & $\nabla^{2} \rho$ & $\rho$ & $\nabla^{2} \rho$ & $\rho$ & $\nabla^{2} \rho$ \\
\hline \multirow[t]{3}{*}{$\overline{\mathrm{Cu}(\mathrm{II})}$} & $(\text { phen })_{2}\left(\mathrm{H}_{2} \mathrm{O}\right)$ & 0.0774 & 0.928 & 0.0653 & 0.0786 & 0.0768 & 0.0927 & 0.0645 & 0.0778 & 0.0363 & 0.0397 \\
\hline & (2-Clip-phen) $\left(\mathrm{H}_{2} \mathrm{O}\right)$ & 0.0669 & 0.0810 & 0.0729 & 0.0894 & 0.0833 & 0.1030 & 0.0332 & 0.0254 & 0.0277 & 0.0227 \\
\hline & (3-Clip-phen) $\left(\mathrm{H}_{2} \mathrm{O}\right)$ & 0.0688 & 0.0828 & 0.0655 & 0.0794 & 0.0727 & 0.0873 & 0.0637 & 0.0770 & 0.0352 & 0.0367 \\
\hline \multirow[t]{3}{*}{$\mathrm{Cu}(\mathrm{II})$} & $(\text { phen })_{2}(\mathrm{OH})$ & 0.0748 & 0.0603 & 0.0328 & 0.0241 & 0.103 & 0.0920 & 0.0860 & 0.0504 & 0.0876 & 0.170 \\
\hline & (2-Clip-phen) (OH) & 0.133 & 0.0522 & 0.152 & 0.0675 & 0.119 & 0.0541 & -- & -- & 0.194 & 0.134 \\
\hline & (3-Clip-phen) (OH) & 0.125 & 0.009 & 0.0700 & 0.0322 & 0.114 & 0.0747 & 0.125 & 0.009 & 0.185 & 0.126 \\
\hline \multirow[t]{3}{*}{$\mathrm{Cu}(\mathrm{II})$} & $(\text { phen })_{2}(\mathrm{OH})^{*}$ & 0.0987 & 0.105 & 0.0306 & 0.0283 & 0.0990 & 0.102 & 0.0817 & 0.0909 & 0.120 & 0.175 \\
\hline & $(2-\mathrm{Clip}-$ phen $)(\mathrm{OH})^{*}$ & 0.0724 & 0.101 & 0.0706 & 0.0867 & 0.0642 & 0.107 & -- & -- & 0.121 & 0.147 \\
\hline & (3-Clip-phen) $(\mathrm{OH})^{*}$ & 0.112 & 0.088 & 0.0445 & 0.0254 & 0.141 & 0.101 & 0.141 & 0.101 & 0.142 & 0.190 \\
\hline
\end{tabular}

*copper complexes with the radical $\mathrm{OH}^{*}$ 
(1) Howell, S. L.; Gordon, K. C. J. Phys. Chem. A 2004, 108, 2536-2544.

(2) Chen, L. X.; Shaw, G. B.; Novozhilova, I.; Liu, T.; Jennings, G.; Attenkofer, K.; Meyer, G. J.; Coppens, P. J. Am. Chem. Soc. 2003, 125, 7022-7034.

(3) Philip Coppens, I. V. N. Int. J. Quantum Chem. 2005, 101, 611-623.

(4) Chen, T.; Greenberg, M. M. J. Am. Chem. Soc. 1998, 120, 3815-3816.

(5) Siddique, Z. A.; Yamamoto, Y.; Ohno, T.; Nozaki, K. Inorg. Chem. 2003, 42, 6366-6378.

(6) Zgierski, M. Z. J. Chem. Phys. 2003, 118, 4045-4051.

(7) Osako, T.; Nagatomo, S.; Kitagawa, T.; Cramer, C.; Itoh, S. J. Biol. Inor. Chem. 2005, 10, 581590.

(8) Wang, X. J.; Wang, W.; Koyama, M.; Kubo, M.; Miyamoto, A. J. Photochem. Photobiol., A 2006, 179, 149-155.

(9) Murphy, G.; Murphy, C.; Murphy, B.; Hathaway, B. J. Chem. Soc. Dalton Trans. 1997, $2653-$ 2600.

(10) King, G.; Gembicky, M.; Coppens, P. Acta Crystallogr., Sect. C: Cryst. Struct. Commun. 2005, 61, M329-M332.

(11) Ruthkosky, M.; Castellano, F. N.; Meyer, G. J. Inorg. Chem. 1996, 35, 6406-6412.

(12) Howell, S. L.; Gordon, K. C. J. Phys. Chem. A 2004, 108, 2536-2544.

(13) Pitie, M.; Boldron, C.; Gornitzka, H.; Hemmert, C.; Donnadieu, B.; Meunier, B. Eur. J. Inorg. Chem. 2003, 528-540. 